\title{
Renormalized squares of Boson fields
}

\author{
L. Accardi* R. Roschin ${ }^{\dagger}$
}

October 20, 2015

\begin{abstract}
The standard renormalization procedure consists in introducing a cut-off and then trying to remove it by some limiting procedure. In the paper [?] a new renormalization technique was introduced based on the idea of renormalizing a closed set of commutation relations and then finding a nontrivial representation for them. In the paper [?] it was proved that, in the case of quadratic fields the new renormalization procedure leads to quadratic field operator which is gamma distributed in the quadratic vacuum (as one would intuitively expect from the "square" of a white noise) and to Meixner or Pascal distributed Poisson fields. It is natural to ask if the same result can be obtained with the usual cut-off and take-limit procedure. In the present paper we prove that the answer to this question is negative. More precisely, we show that, independently of the choice of the cutoff (cf. section 7), if a quadratic field admits a limit in the sense of mixed moments, then this limit will be Gaussian distributed in the vacuum and consequently the associated Poisson fields will have a Poisson distribution.
\end{abstract}

\section{Introduction}

The problem of defining renormalized powers of quantum fields has motivated several investigations [?, ?]. The Wilson expansion and

\footnotetext{
*Centro Vito Volterra, Università di Roma Tor Vergata, accardi@volterra.mat.uniroma2.it

†Steklov Mathematical Institute, Gubkin St.8, GSP-1, 117966, Moscow, Russia, roschin@mi.ras.ru
} 
the Zimmermann product were developed for this purpose. From the point of view of rigorous mathematical results we mention the paper [?] where it is proved that the square of the usual time zero, scalar, Fock, Klein-Gordon field on $\mathbb{R}^{d}$, cannot correspond to a self-adjoint operator acting on the same Fock space of the field unless $d=1$.

The proof of Segal's result exploits the canonical commutation relations (CCR) to prove that, if such a self-adjoint operator would exist, then the associated 1-parameter unitary group should induce a 1-parameter automorphism group of the Weyl algebra which, by construction, should be inner. On the other hand, the explicit form of this 1-parameter automorphism group shows that it is the second quantization of a 1-parameter family of operators acting on $L^{2}\left(\mathbb{R}^{d}\right)$. This allows, using the explicit form of the spectral function of the Klein-Gordon field $\left[\left(m+k^{2}\right)^{-1 / 2}\right]$, to prove that only in the case $d=1$ the conditions of Shale's theorem on the unitary implementability of automorphisms of the Weyl algebra [?] can be satisfied.

On the other hand Segal's result does not exclude the possibility of a coherent definition of the renormalized square of a Fock boson field in a Hilbert space different from the Fock space where the field itself acts.

The natural idea of using a Bogolyubov transformation to diagonalize a quadratic expression in the field was analyzed in [?] where it was shown that the set of parameters for which such a Bogolyubov transformation exists does not include the critical value 2 which is precisely the one, corresponding to the square of field (classical white noise) one was trying to define.

In 1999 Accardi, Lu and Volovich [?] proposed a different approach to the definition of the renormalized square of a Boson Fock field on $\mathbb{R}^{d}$ ( $d$-dimensional white noise), based on the following idea: instead of renormalizing the action of the hypothetical "square of the field" on the Fock space of the 1-st powers of the field, they renormalized the commutation relations of the second powers of the field and proved constructively that a Fock representation for them exists.

This result gave rise to a rather impetuous development [?], [?], [?], [?], [?], [?], [?] from which it emerged that the realizations of the RSWN are representations of the current algebra over $\operatorname{sl}\left(2 ; \mathbb{R}^{d}\right)$ and that, just like the vacuum expectations of linear combinations of the first order fields produce the usual Gaussian and Poisson distributions, the vacuum expectations of linear combinations of second order fields produce exactly all the remaining three family of distributions in the 
Meixner classes (i.e. Pascal, gamma and Meixner).

It is worth emphasizing that, from a result of P. Sniady [?], it follows that even in dimension 1 the renormalized square of white noise in the sense of [?] cannot live on the same Fock space of the usual first order field. This proves that, even in dimension 1, the renormalization procedure of Accardi, Lu, Volovich leads to field operators different from those constructed by Segal.

In view of all these developments a natural question is to ask wether the renormalized square of white noise (RSWN) can be obtained with a procedure nearer to the usual approaches to renormalization theory (see, for example, [?]), namely:

(i) introducing a cut-off

(ii) possibly compensating in some way divergent quantities

(iii) using a limit procedure to eliminate the cut-off

In the present paper we prove that the answer to the above question is negative. More precisely, we prove that, for a large class of natural cut-off functions, if the cut-off can be removed by a converging limiting procedure then the limit field is necessarily gaussian.

Our starting point is the family of quadratic expressions in the field operators, which can be symbolically written in the form :

$$
H=\int_{\mathbb{R}^{d}} d p\left(\omega(p)\left(a_{p}^{2}+\left(a_{p}^{+}\right)^{2}\right)+\nu(p) a_{p}^{+} a_{p}\right)
$$

where $a_{p}^{+}$and $a_{p}$ are Bose creation and annihilation operators in the Fock representation. Since $a_{p}$ is an operator-valued distribution and the multiplication of distributions is not uniquely defined, one has to specify a framework to give a meaning to expressions like (??) and, as already mentioned before, several procedures have been proposed in the literature in order to achieve this goal.

A natural way of dealing with the multiplication of distributions is a regularization. The naive idea is to replace $a_{p}$ by an expression of the form

$$
a_{p, \varepsilon}=\int_{\mathbb{R}^{d}} d k \delta_{\varepsilon}(k) a_{p+k}
$$

where $\delta_{\varepsilon}$ is a delta-sequence, that is a sequence of smooth functions such that $\forall f \in \mathcal{S}\left(\mathbb{R}^{d}\right)$

$$
\lim _{\varepsilon \rightarrow 0} \int_{\mathbb{R}^{d}} d k \delta_{\varepsilon}(k) f(k)=f(0)
$$


Since $a_{p, \varepsilon}$ is a well-defined operator for a wide class of test functions $\delta_{\varepsilon}$, one can replace $a_{p}$ by $a_{p, \varepsilon}$ in (??), and take the limit $\varepsilon \rightarrow 0$ in some sense to be specified. However this naive procedure leads to the divergence of the commutator $\left[a_{p, \varepsilon}^{2},\left(a_{k, \varepsilon}^{+}\right)^{2}\right]$ in the limit $\varepsilon \rightarrow 0$ hence to the necessit of a renormalization procedure. The usual renormalization procedure consists in defining a regularized $a_{p, \varepsilon}$ through the prescription:

$$
a_{p, \varepsilon}=\varepsilon^{A} \int_{\mathbb{R}^{d}} d p \delta_{\varepsilon}(p) a_{p}
$$

and, in analogy with the classical central limit theorems, we prove (cf. Lemma (5) below) that the only way to obtain a limit which is not identically zero or infinity is to choose $A=d / 2$. Then we prove the main result of the present paper, namely: independently of the special form of the delta-sequence $\delta_{\varepsilon}(p)$ the expressions in $a_{p, \varepsilon}$ converge, in sense of distribution mixed moments (correlators), to the standard Bose Fock creation and annihilation operators.

The plan of the present paper is the following. In Section (2) we introduce the basic notations and the smeared quadratic fields. The structure of the associated Lie algebra is introduced in Section (3). In Section (4) we introduce a particular regularization and study its main properties. The Gaussianity of the limit of the Lie algebra of smeared quadratic fields, with respect to this regularization, is established in Sections (5) and (6). This means that, in the limit $\varepsilon \rightarrow 0$, the squares of the quadratic Bose operators converge, in the sense of correlators, to a Bose Fock field.

Finally in Section (7) we prove the robustnsess of our main result, i.e. the Gaussianity of the limit, with respect to the choice of the regularization of the $\delta$-function within a quite general class which includes the special choice introduced in Section (4).

\section{Smeared quadratic fields}

We use the following notations: $\mathcal{S}\left(\mathbb{R}^{d}\right)$ denotes the Schwartz space, $\mathcal{S}^{\prime}\left(\mathbb{R}^{d}\right)$ - the space of Schwartz distributions (see, for example, [?]); $\langle\cdot, \cdot\rangle$ denotes the scalar product either in the Bose Fock space, or in any $N$-particle subspaces $\mathcal{H}_{B}^{N}$ (see Definition ?? below), in particular, if $\phi, \psi \in L^{2}\left(\mathbb{R}^{d}\right),\langle\phi, \psi\rangle=\int d k \phi(k) \psi(k)$.

All indices $k, p$ are $d$-dimensional and, when the domain of integration is not specified in an integral, this is understood to be $\mathbb{R}^{d}$. 
Definition 1 A Boson Fock field on $\mathbb{R}^{d}$ is a field $a_{k}, a_{k^{\prime}}^{+}$together with an expectation value \langle\rangle such that

$$
\begin{gathered}
\left\langle a_{k}^{+} a_{k^{\prime}}\right\rangle=0 \\
\left.\left\langle a_{k_{1}}^{\varepsilon_{1}} \ldots a_{k_{2 n}}^{\varepsilon_{n}}\right\rangle=\sum_{\text {All pair partitions }\left(l_{i}, r_{i}\right)}^{\varepsilon_{1}} \text { of } a_{k_{1}}^{\varepsilon_{1}} \ldots a_{k_{n}}^{\varepsilon_{n}}\right\rangle=0, \text { if } n \text { is odd, } \\
\left\langle a_{k_{k_{2 n}}}^{\varepsilon_{l_{1}}} a_{k_{r_{1}}}^{\varepsilon_{r_{1}}}\right\rangle \ldots\left\langle a_{k_{l_{n}}}^{\varepsilon_{l_{n}}} a_{k_{r_{n}}}^{\varepsilon_{r_{n}}}\right\rangle
\end{gathered}
$$

where $a^{\varepsilon}$ means $a^{+}$or $a$.

Remark. The boson Fock property is equivalent to the condition (see [?], 2.11):

$$
\left\langle e^{i t\left(A_{\phi}^{+}+A_{\phi}\right)}\right\rangle=e^{\frac{1}{2} t^{2}\langle\phi, \phi\rangle}
$$

Boson Fock fields are realized on Boson Fock spaces.

Definition 2 The Bose Fock space over $L^{2}\left(\mathbb{R}^{d}\right)$ is

$$
\mathcal{F}_{B}\left(L^{2}\left(\mathbb{R}^{d}\right)\right):=\bigoplus_{n=0}^{\infty} \mathcal{H}_{B}^{n}
$$

where $\mathcal{H}_{B}^{n}$ is the space of symmetric square integrable functions of $n$ arguments, that is, complex-valued function $v_{n}\left(x_{1}, \ldots x_{n}\right) \in \mathcal{H}_{B}^{N}$ if and only if

$$
\int d x_{1} \ldots d x_{n}\left|v_{n}\left(x_{1}, \ldots x_{n}\right)\right|^{2}<\infty
$$

and for any $1 \leq i<j \leq n$

$$
v_{n}\left(x_{1}, \ldots, x_{i}, \ldots, x_{j}, \ldots, x_{n}\right)=v_{n}\left(x_{1}, \ldots, x_{j}, \ldots, x_{i}, \ldots, x_{n}\right)
$$

Any vector $V$ from the Bose Fock space can be represented as

$$
V=\left(v_{0}, v_{1}\left(x_{1}\right), v_{2}\left(x_{1}, x_{2}\right), \ldots, v_{n}\left(x_{1}, x_{2}, \ldots, x_{n}\right), \ldots\right)
$$

The scalar product of two vectors $V, W$ is given by:

$$
\langle V, W\rangle=\sum_{n=0}^{\infty}\left\langle v_{n}, w_{n}\right\rangle
$$

where the scalar product in $\mathcal{H}_{B}^{n}$ is

$$
\left\langle v_{n}, w_{n}\right\rangle=\int d x_{1} \ldots d x_{n} v_{n}\left(x_{1}, \ldots, x_{n}\right) w_{n}\left(x_{1}, \ldots, x_{n}\right)
$$

The vector $\psi_{0}=(1,0,0, \ldots)$ is called the vacuum vector. Vectors with $v_{n}=0$, except for at most one $n$, are called number or $n$-particle vectors. 
Definition 3 Consider an n-particle vector:

$$
V_{n}=\left(0,0, \ldots, 0, v_{n}\left(x_{1}, x_{2}, \ldots, x_{n}\right), 0 \ldots\right)
$$

The Bose creation operator $a^{+}(f)$ is defined by its action on $V_{n}$ :

$$
a^{+}(f) V_{n}=U_{n+1}
$$

where $U_{n+1}$ is the $(n+1)$-particle vector given by

$$
u_{n+1}\left(x_{1}, x_{2}, \ldots x_{n+1}\right)=\sqrt{n} \sum_{i=1}^{n+1} f\left(x_{i}\right) v_{n}\left(x_{1}, \ldots, x_{i-1}, x_{i+1}, \ldots x_{n+1}\right)
$$

The action of the Bose annihilation operator $a(f)$ is defined by

$$
\begin{gathered}
a(f) \psi_{0}=0 \\
a(f) V_{n}=W_{n-1} ; \quad \text { if } n \geq 1
\end{gathered}
$$

where $W_{n-1}$ is the $(n-1)$-particle vector given by

$$
w_{n-1}\left(x_{1}, x_{2}, \ldots x_{n+1}\right)=\sqrt{n-1} \sum_{i=1}^{n} \int d x_{i} f\left(x_{i}\right) v_{n}\left(x_{1}, x_{2}, \ldots x_{n}\right)
$$

We extend the definition of $a$ and $a^{+}$to all finite linear combinations of number vectors by linearity.

We use the following notation:

$$
a(f)=\int a_{k} f(k) d k ; a^{+}(f)=\int a_{k}^{+} f(k) d k
$$

$a_{k}^{+}$and $a_{k}$ are called the Bose creation and annihilation operatorvalued distributions. When no confusion is possible, the pair $a_{k}^{+}, a_{k}$ is simply called a "Bose Fock field".

It is easy to check that $a(f)$ and $a^{+}(g)$ satisfy the following commutation relations which define the CCR (or Heisenberg) Lie algebra over $\mathcal{S}\left(\mathbb{R}^{d}\right)$ :

$$
\left[a(f), a^{+}(g)\right]=\langle f, g\rangle
$$

or, in distributions notation:

$$
\left[a_{p}, a_{k}^{+}\right]=\delta(p-k)
$$


Definition 4 For $f, g, h \in L^{2}\left(\mathbb{R}^{d}\right)$ define the smeared quadratic fields by:

$$
\begin{aligned}
B_{f}^{+} & :=\int f\left(k_{1}, k_{2}\right) a_{k_{1}}^{+} a_{k_{2}}^{+} d k_{1} d k_{2} \\
B_{g}^{-} & :=\int \bar{g}\left(p_{1}, p_{2}\right) a_{p_{1}} a_{p_{2}} d p_{1} d p_{2}
\end{aligned}
$$

where the test functions are supposed to be symmetric

$$
\begin{gathered}
f\left(k_{1}, k_{2}\right)=f\left(k_{2}, k_{1}\right) ; \quad g\left(p_{1}, p_{2}\right)=g\left(p_{2}, p_{1}\right) \\
N_{h}:=\int h\left(k_{1}, k_{2}\right) a_{k_{1}}^{+} a_{k_{2}} d k_{1} d k_{2}
\end{gathered}
$$

notice that $h$ is not supposed to be symmetric.

\section{The quadratic Lie algebra}

Definition 5 For $f, g, h \in L^{2}\left(\mathbb{R}^{d}\right)$ define:

$$
\begin{aligned}
(f \diamond g)\left(p_{1}, p_{2}\right) & :=\int d k f\left(p_{1}, k\right) g\left(k, p_{2}\right) \\
\operatorname{Tr} f & :=\int d k f(k, k)
\end{aligned}
$$

Lemma 1 The smeared quadratic fields satisfy the following commutation relations:

$$
\begin{gathered}
{\left[B_{g}^{-}, B_{f}^{+}\right]=4 N_{f \diamond \bar{g}}+2 \operatorname{Tr}(\bar{g} \diamond f)} \\
{\left[N_{h}, B_{f}^{+}\right]=2 B_{h \diamond f}^{+}} \\
{\left[N_{h}, B_{f}^{-}\right]=-2 B_{h \diamond \bar{g}}^{-}} \\
{\left[N_{f}, N_{g}\right]=N_{f \diamond g-g \diamond f}=N_{[f, g]_{\diamond}}} \\
{\left[B_{f}^{+}, B_{g}^{+}\right]=\left[B_{f}^{-}, B_{g}^{-}\right]=0}
\end{gathered}
$$

Remark. The above result shows that the quadratic fields form a closed Lie algebra, called the quadratic Lie algebra. Notice the analogy between this Lie algebra and the Lie algebra of the renormalized square of white noise [?]: the only difference between the two is that here the pointwise multiplication among test functions is replaced by the $\diamond-$ product which is not commutative. 
Proof. We have:

$$
\left[B_{g}^{-}, B_{f}^{+}\right]=\int d k_{1} d k_{2} d p_{1} d p_{2} \bar{g}\left(k_{1}, k_{2}\right) f\left(p_{1}, p_{2}\right)\left[a_{k_{1}} a_{k_{2}}, a_{k_{1}}^{+} a_{k_{2}}^{+}\right]
$$

Moreover

$$
\begin{gathered}
{\left[a_{k_{1}} a_{k_{2}}, a_{p_{1}}^{+} a_{p_{2}}^{+}\right]=\left[a_{k_{1}} a_{k_{2}}, a_{p_{1}}^{+}\right] a_{p_{2}}^{+}+a_{p_{1}}^{+}\left[a_{k_{1}} a_{k_{2}}, a_{p_{2}}^{+}\right]} \\
=a_{k_{1}}\left[a_{k_{2}}, a_{p_{1}}^{+}\right] a_{p_{2}}^{+}+\left[a_{k_{1}}, a_{p_{1}}^{+}\right] a_{k_{2}} a_{p_{2}}^{+}+a_{p_{1}}^{+} a_{k_{1}}\left[a_{k_{2}}, a_{p_{2}}^{+}\right]+a_{p_{1}}^{+}\left[a_{k_{1}}, a_{p_{2}}^{+}\right] a_{k_{2}} \\
=a_{k_{1}} a_{p_{2}}^{+} \delta\left(k_{2}-p_{1}\right)+\delta\left(k_{1}-p_{1}\right) a_{k_{2}} a_{p_{2}}^{+}+a_{p_{1}}^{+} a_{k_{1}} \delta\left(k_{2}-p_{2}\right)+a_{p_{1}}^{+} a_{k_{2}} \delta\left(k_{1}-p_{2}\right) \\
=a_{p_{2}}^{+} a_{k_{1}} \delta\left(k_{2}-p_{1}\right)+a_{p_{2}}^{+} a_{k_{2}} \delta\left(k_{1}-p_{1}\right)+a_{p_{1}}^{+} a_{k_{1}} \delta\left(k_{2}-p_{2}\right)+a_{p_{1}}^{+} a_{k_{2}} \delta\left(k_{1}-p_{2}\right)+ \\
+\delta\left(k_{1}-p_{2}\right) \delta\left(k_{2}-p_{1}\right)+\delta\left(k_{1}-p_{1}\right) \delta\left(k_{2}-p_{2}\right)
\end{gathered}
$$

After evaluation of the $\delta$-function the integrands of the terms with a single $\delta$-function are

$$
\begin{aligned}
& \bar{g}\left(k_{1}, k_{2}\right) f\left(p_{2}, p_{1}\right) a_{p_{2}}^{+} a_{k_{1}} \\
& \bar{g}\left(k_{1}, k_{2}\right) f\left(k_{1}, p_{2}\right) a_{p_{2}}^{+} a_{k_{2}} \\
& \bar{g}\left(k_{1}, k_{2}\right) f\left(p_{1}, k_{2}\right) a_{p_{1}}^{+} a_{k_{1}} \\
& \bar{g}\left(k_{1}, k_{2}\right) f\left(p_{1}, k_{1}\right) a_{p_{1}}^{+} a_{k_{2}}
\end{aligned}
$$

and, using the symmetry of $f, g$ they become

$$
\begin{aligned}
& \bar{g}\left(k_{1}, k_{2}\right) f\left(k_{2}, p_{2}\right) a_{p_{2}}^{+} a_{k_{1}} \\
& \bar{g}\left(k_{2}, k_{1}\right) f\left(k_{1}, p_{2}\right) a_{p_{2}}^{+} a_{k_{2}} \\
& \bar{g}\left(k_{1}, k_{2}\right) f\left(k_{2}, p_{1}\right) a_{p_{1}}^{+} a_{k_{1}} \\
& \bar{g}\left(k_{2}, k_{1}\right) f\left(k_{1}, p_{1}\right) a_{p_{1}}^{+} a_{k_{2}}
\end{aligned}
$$

Changing variables in (??): $k_{1} \rightarrow k_{2} ; k_{2} \rightarrow k_{1}$ we obtain

$$
\bar{g}\left(k_{1}, k_{2}\right) f\left(k_{2}, p_{2}\right) a_{p_{2}}^{+} a_{k_{1}}
$$

with a similar change of variables in (??): $k_{2} \rightarrow p_{1} ; p_{1} \rightarrow p_{2}$ we obtain

$$
\bar{g}\left(k_{1}, k_{2}\right) f\left(k_{2}, p_{2}\right) a_{p_{2}}^{+} a_{k_{1}}
$$

Similarly in (??) changing variables:

$$
k_{1} \rightarrow p_{1}, \quad k_{2} \rightarrow p_{2} ; \quad k_{2} \rightarrow k_{1}
$$


we obtain

$$
\bar{g}\left(k_{1}, k_{2}\right) f\left(k_{2}, p_{2}\right) a_{p_{2}}^{+} a_{k_{1}}
$$

and this gives:

$4 \int d p_{2} d k_{1} a_{p_{2}}^{+} a_{k_{1}} \int d k_{2} \bar{g}\left(k_{1}, k_{2}\right) f\left(k_{2}, p_{2}\right)=4 \int d p_{2} d k_{1} a_{p_{2}}^{+} a_{k_{1}}(f \diamond \bar{g})\left(k_{1}, p_{2}\right)=4 N_{\bar{g} \diamond f}$

The integrands of the terms with two $\delta$-functions are

$$
g\left(k_{1}, k_{2}\right) f\left(k_{2}, k_{1}\right) ; \quad g\left(k_{1}, k_{2}\right) f\left(k_{1}, k_{2}\right)
$$

and this gives the second term in the right hand side of (??):

$$
2 \int d k_{1} d k_{2} \bar{g}\left(k_{1}, k_{2}\right) f\left(k_{2}, k_{1}\right)=2 \operatorname{Tr} \bar{g} \diamond f
$$

To prove (??) notice that

$$
\left[N_{h}, B_{f}^{+}\right]=\int d k_{1} d k_{2} \int d p_{1} d p_{2} h\left(k_{1}, k_{2}\right) f\left(p_{1}, p_{2}\right)\left[a_{k_{1}}^{+} a_{k_{2}}, a_{p_{1}}^{+} a_{p_{2}}^{+}\right]
$$

Now

$$
\begin{gathered}
{\left[a_{k_{1}}^{+} a_{k_{2}}, a_{p_{1}}^{+} a_{p_{2}}^{+}\right]=\left[a_{k_{1}}^{+} a_{k_{2}}, a_{p_{1}}^{+}\right] a_{p_{2}}^{+}+a_{p_{1}}^{+}\left[a_{k_{1}}^{+} a_{k_{2}}, a_{p_{2}}^{+}\right]=} \\
=a_{k_{1}}^{+}\left[a_{k_{2}}, a_{p_{1}}^{+}\right] a_{p_{2}}^{+}+a_{p_{1}}^{+} a_{k_{1}}^{+}\left[a_{k_{2}}, a_{p_{2}}^{+}\right]= \\
=a_{k_{2}}^{+} a_{p_{2}}^{+} \delta\left(k_{2}-p_{1}\right)+a_{p_{1}}^{+} a_{k_{1}}^{+} \delta\left(k_{2}-p_{2}\right)
\end{gathered}
$$

The two corresponding integrands are

$$
\begin{gathered}
h\left(k_{1}, k_{2}\right) f\left(k_{2}, p_{2}\right) a_{k_{1}}^{+} a_{p_{2}}^{+} \\
h\left(k_{1}, k_{2}\right) f\left(p_{1}, k_{2}\right) a_{p_{1}}^{+} a_{k_{1}}^{+}=h\left(k_{1}, k_{2}\right) f\left(k_{2}, p_{1}\right)+a_{p_{1}}^{+} a_{k_{1}}^{+}
\end{gathered}
$$

changing variables in the second term: $p_{1} \rightarrow k_{1}, k_{1} \rightarrow p_{2}$ one obtains

$$
h\left(k_{1}, k_{2}\right) f\left(k_{2}, p_{2}\right) a_{k_{1}}^{+} a_{p_{2}}^{+}
$$

and the sum of the two integrands becomes

$$
2 h\left(k_{1}, k_{2}\right) f\left(k_{2}, p_{2}\right) a_{k_{1}}^{+} a_{p_{2}}^{+}
$$

Integrating one obtains:

$$
2 \int d k_{1} d p_{2} a_{k_{1}}^{+} a_{p_{2}}^{+} \int d k_{2} h\left(k_{1}, k_{2}\right) f\left(k_{2}, p_{2}\right)=2 \int d k_{1} d p_{2} a_{k_{1}}^{+} a_{p_{2}}^{+}(h \diamond f)\left(k_{1}, p_{2}\right)=2 B_{h \diamond f}^{+}
$$


Similarly, one can get (??).

Finally,

$$
\left[N_{f}, N_{g}\right]=\int d k_{1} d k_{2} \int d p_{1} d p_{2} f\left(k_{1}, k_{2}\right) g\left(p_{1}, p_{2}\right)\left[a_{k_{1}}^{+} a_{k_{2}}, a_{p_{1}}^{+} a_{p_{2}}\right]
$$

Since

$$
\begin{aligned}
{\left[a_{k_{1}}^{+} a_{k_{2}}, a_{p_{1}}^{+} a_{p_{2}}\right] } & =\left[a_{k_{1}}^{+} a_{k_{2}}, a_{p_{1}}^{+}\right] a_{p_{2}}+a_{p_{1}}^{+}\left[a_{k_{1}}^{+} a_{k_{2}}, a_{p_{2}}\right]=a_{k_{1}}^{+}\left[a_{k_{2}}, a_{p_{1}}^{+}\right] a_{p_{2}}+a_{p_{1}}^{+}\left[a_{k_{1}}^{+}, a_{p_{2}}\right] a_{k_{2}}= \\
& =\delta\left(k_{2}-p_{1}\right) a_{k_{1}}^{+} a_{p_{2}}-\delta\left(p_{2}-k_{1}\right) a_{p_{1}}^{+} a_{k_{2}}
\end{aligned}
$$

it follows that

$$
\begin{aligned}
& \quad\left[N_{f}, N_{g}\right]= \\
& =\int d k_{1} d p_{2} a_{k_{1}}^{+} a_{p_{2}} \int f\left(k_{1}, k_{2}\right) g\left(k_{2}, p_{2}\right) d k_{2}-\int d p_{1} d k_{2} a_{p_{1}}^{+} a_{k_{2}} \int g\left(p_{1}, k_{1}\right) f\left(k_{1}, k_{2}\right) d k_{1}= \\
& =N_{f \diamond g}-N_{g \diamond f}
\end{aligned}
$$

and this proves (??). Eq. (??) is obvious.

\section{A particular regularization of the $\delta$ - function and some of its properties}

Lemma 2 Define, for $\varepsilon \neq 0$

$$
\delta_{\varepsilon}(x):=\frac{1}{\pi^{d / 2} \varepsilon^{d}} e^{-\frac{x^{2}}{\varepsilon^{2}}} ; \quad x \in \mathbb{R}^{d}
$$

where $x^{2}=x_{1}^{2}+\cdots+x_{d}^{2}$. Then as $\varepsilon \rightarrow 0 \delta_{\varepsilon}(x) \rightarrow \delta(x)$ in $\mathcal{S}^{\prime}\left(\mathbb{R}^{d}\right)$.

Proof. For any $\varphi \in \mathcal{S}\left(\mathbb{R}^{d}\right)$,

$$
\int \delta_{\varepsilon}(x) \varphi(x) d x=\int \frac{1}{\pi^{d / 2} \varepsilon^{d}} e^{-\frac{x^{2}}{\varepsilon^{2}}} \varphi(x) d x=\frac{1}{\pi^{d / 2}} \int e^{-y^{2}} \varphi(\varepsilon y) d y
$$

Here we denote $\varepsilon y:=\left(\varepsilon y_{1}, \ldots, \varepsilon y_{d}\right)$. As $\varepsilon \rightarrow 0$ this converges to

$$
\frac{\varphi(0)}{\pi^{d / 2}} \int e^{-y^{2}} d y=\varphi(0)
$$

where we used the identity

$$
\int e^{-y^{2}} d y=\pi^{d / 2}
$$


Lemma 3 Let $\delta_{\varepsilon}(x)$ be as in (??). Then

$$
\delta_{\varepsilon}(x)^{2}=\frac{1}{(2 \pi)^{d / 2} \varepsilon^{d}} \delta_{\frac{\varepsilon}{\sqrt{2}}}(x)
$$

Proof.

$$
\begin{aligned}
& \delta_{\varepsilon}(x)^{2}=\frac{1}{\pi^{d} \varepsilon^{2 d}} e^{-\frac{2 x^{2}}{\varepsilon^{2}}}= \\
& \quad=\frac{1}{\pi^{d / 2}(\varepsilon / \sqrt{2})^{d} \cdot \pi^{d / 2} \cdot(\sqrt{2} \varepsilon)^{d}} e^{-\frac{x^{2}}{(\varepsilon / \sqrt{2})^{2}}}=\frac{1}{(2 \pi)^{d / 2} \varepsilon^{d}} \delta_{\frac{\varepsilon}{\sqrt{2}}}(x)
\end{aligned}
$$

Lemma 4 For any $m \geq 1, f \in \mathcal{S}\left(\mathbb{R}^{m}\right)$

$$
\begin{gathered}
\lim _{\varepsilon \rightarrow 0} \varepsilon^{d} \int d x_{1} \ldots d x_{m} f\left(x_{1}, \ldots, x_{m}\right) \delta_{\varepsilon}\left(x_{1}-x_{2}\right) \delta_{\varepsilon}\left(x_{2}-x_{3}\right) \ldots \delta_{\varepsilon}\left(x_{m}-x_{1}\right)= \\
=\frac{1}{(2 \pi)^{(m-1) d / 2}} \int_{\mathbb{R}^{d}} f(x, x, \ldots, x) d x
\end{gathered}
$$

Proof. With the change of variables:

$$
\varepsilon y_{1}=\left(x_{2}-x_{1}\right), \ldots, \varepsilon y_{m-1}=\left(x_{m}-x_{m-1}\right)
$$

one has:

$$
\begin{gathered}
I(\varepsilon)=\varepsilon^{d} \int_{\mathbb{R}^{d}} d x_{1} \ldots d x_{m} f\left(x_{1}, \ldots, x_{m}\right) \delta_{\varepsilon}\left(x_{1}-x_{2}\right) \delta_{\varepsilon}\left(x_{2}-x_{3}\right) \ldots \delta_{\varepsilon}\left(x_{m}-x_{1}\right)= \\
=\frac{1}{\pi^{m d / 2}} \int d x_{1} d y_{1} \ldots d y_{m-1} f\left(x_{1}, x_{1}+\varepsilon y_{1} \ldots, x_{1}+\varepsilon y_{1}+\cdots+\varepsilon y_{m-1}\right) \times \\
\times e^{-y_{1}^{2}-y_{2}^{2}-\cdots-y_{m-1}^{2}-\left(y_{1}+\cdots+y_{m-1}\right)^{2}}
\end{gathered}
$$

By dominated convergence and computing the gaussian integral, (??) follows.

\section{The CCR algebra as diagonal limit of the quadratic algebra}

Definition 6 For $f \in L^{2}\left(\mathbb{R}^{2 d}\right)$, define:

$$
f_{\varepsilon}\left(p_{1}, p_{2}\right)=f\left(p_{1}, p_{2}\right) \delta_{\varepsilon}\left(p_{1}-p_{2}\right)
$$


Definition 7 We fix an embedding $S: \mathcal{S}\left(\mathbb{R}^{d}\right) \rightarrow L^{2}\left(\mathbb{R}^{2 d}\right)$ with the following properties: if $f=S(\phi)$, then

$$
f(p, p)=\phi(p)
$$

and $\exists \mu>0$, such that $f$ is smooth in the $\mu$-neighborhood of the diagonal: $p_{1}=p_{2}$. For $\phi \in \mathcal{S}\left(\mathbb{R}^{d}\right), f=S(\phi)$ and $f_{\varepsilon}$ given by (??), define:

$$
\begin{gathered}
B_{\phi, \varepsilon}^{+}=c \varepsilon^{d / 2} B_{f_{\varepsilon}}^{+} \\
B_{\phi, \varepsilon}^{-}=c \varepsilon^{d / 2} B_{f_{\varepsilon}}^{-} \\
N_{\phi, \varepsilon}=N_{f_{\varepsilon}}
\end{gathered}
$$

where $c=2^{(d / 4-1 / 2)} \pi^{d / 4}$.

Notice that different embeddings for different functions are allowed.

Definition 8 Consider $a$. The operator $A$ is said to be a weak limit, on the number vectors, of the sequence of operators $A_{n}$ if, for any pair of number vectors $V_{1}, V_{2}$, one has:

$$
\lim _{n \rightarrow \infty}\left\langle V_{1}, A_{n} V_{2}\right\rangle=\left\langle V_{1}, A V_{2}\right\rangle
$$

In this case we write:

$$
\mathrm{w}_{n \rightarrow \infty} A_{n}=A
$$

The following theorem proves that in the limit, as $\varepsilon \rightarrow 0$, the quadratic Lie algebra becomes the usual CCR Lie algebra over $L^{2}\left(\mathbb{R}^{d}\right)$.

Theorem 1 Suppose $\phi, \psi \in \mathcal{S}\left(\mathbb{R}^{d}\right)$. Then

$$
\begin{aligned}
& \underset{\varepsilon \rightarrow 0}{\mathrm{w} .} \lim _{\varepsilon \rightarrow \varepsilon}\left[B_{\phi, \varepsilon}^{-}, B_{\psi, \varepsilon}^{+}\right]=\langle\phi, \psi\rangle \\
& \mathrm{w}_{\varepsilon \rightarrow 0} \lim _{\varepsilon \rightarrow \varepsilon}\left[B_{\phi, \varepsilon}^{+}, N_{\psi, \varepsilon}\right]=0 \\
& \mathrm{w}_{\varepsilon \rightarrow 0} \lim _{\phi, \varepsilon}\left[B_{\phi, \varepsilon}^{-}, N_{\psi, \varepsilon}\right]=0
\end{aligned}
$$

Moreover these limits don't depend on the embeddings $S_{1}, S_{2}$.

Proof. Denote $f=S_{1}(\phi), g=S_{2}(\psi)$. Let us prove (??). From Definition ?? we deduce:

$$
\left[B_{\phi, \varepsilon}^{-}, B_{\psi, \varepsilon}^{+}\right]=c^{2} \varepsilon^{d}\left[B_{f_{\varepsilon}}^{-}, B_{g_{\varepsilon}}^{+}\right]
$$


Using Lemma ?? we have

$$
c^{2} \varepsilon^{d}\left[B_{f_{\varepsilon}}^{-}, B_{g_{\varepsilon}}^{+}\right]=4 c^{2} \varepsilon^{d} N_{f_{\varepsilon} \diamond g_{\varepsilon}}+2 c^{2} \varepsilon^{d} \operatorname{Tr} f_{\varepsilon} \diamond g_{\varepsilon}
$$

Denote $T_{2}:=2 c^{2} \varepsilon^{d} \operatorname{Tr} f_{\varepsilon} \diamond g_{\varepsilon}$. We have

$$
T_{2}=2 c^{2} \varepsilon^{d} \int d k_{1} d k_{2} f\left(k_{1}, k_{2}\right) g\left(k_{1}, k_{2}\right)\left(\delta_{\varepsilon}\left(k_{1}-k_{2}\right)\right)^{2}
$$

Using Lemma ?? and dominated convergence we obtain:

$$
T_{2}=\frac{2 c^{2}}{(2 \pi)^{d / 2}} \int d k_{1} d k_{2} f\left(k_{1}, k_{2}\right) g\left(k_{1}, k_{2}\right) \delta_{\varepsilon / \sqrt{2}}\left(k_{1}-k_{2}\right)
$$

Note, that $\frac{2 c^{2}}{(2 \pi)^{d / 2}}=1$. Since $f$ and $g$ are smooth in some neighborhood of the line $k_{1}-k_{2}=0$, we can use Lemma ??. We obtain:

$$
\begin{aligned}
\lim _{\varepsilon \rightarrow 0} 2 c^{2} \varepsilon^{d} \operatorname{Tr} f_{\varepsilon} \diamond g_{\varepsilon}=\lim _{\varepsilon \rightarrow 0} T_{2}= \\
\quad=\int d k f(k, k) g(k, k)=\int d k \phi(k) \psi(k)=\langle\phi, \psi\rangle
\end{aligned}
$$

Denote $T_{1}:=4 c^{2} \varepsilon^{d} N_{f_{\varepsilon} \diamond g_{\varepsilon}}$. We have:

$$
T_{1}:=4 c^{2} \varepsilon^{d} \int d p_{1} d p_{2} a_{p_{1}}^{+} a_{p_{2}} \int d k f\left(p_{1}, k\right) g\left(k, p_{2}\right) \delta_{\varepsilon}\left(p_{1}-k\right) \delta_{\varepsilon}\left(k-p_{2}\right)
$$

We claim that $\mathrm{w}_{\varepsilon \rightarrow 0} \lim _{1} T_{1}=0$. Intuitively, our claim is motivated by the fact that, as $\varepsilon \rightarrow 0, \delta_{\varepsilon}(p) \rightarrow \delta(p)$ therefore one expects that:

$$
\begin{array}{r}
T_{1} \sim 4 c^{2} \varepsilon^{d} \int d p_{1} d p_{2} d k a_{p_{1}}^{+} a_{p_{2}} f\left(p_{1}, k\right) g\left(k, p_{2}\right) \delta\left(p_{1}-k\right) \delta\left(k-p_{2}\right)= \\
=4 c^{2} \varepsilon^{d} \int d k a_{k}^{+} f(k, k) g(k, k)=4 c^{2} \varepsilon^{d} N(f(\cdot, \cdot) g(\cdot, \cdot)) \rightarrow 0
\end{array}
$$

We will prove that this is indeed the case. Consider two number vectors $V_{1}, V_{2} \in \mathcal{F}_{B}\left(L^{2}\left(\mathbb{R}^{d}\right)\right)$ of the form

$$
\begin{aligned}
& V_{1}=\left(0,0, \ldots, 0, v_{1}\left(q_{1}, q_{2}, \ldots, q_{N}\right), 0, \ldots\right) \\
& V_{2}=\left(0,0, \ldots, 0, v_{2}\left(q_{1}, q_{2}, \ldots, q_{M}\right), 0, \ldots\right)
\end{aligned}
$$

where $v_{i}$ are smooth functions. If $N \neq M$, then $\left\langle V_{1}, T_{1} V_{2}\right\rangle=0$. Otherwise,

$\left\langle V_{1}, T_{1} V_{2}\right\rangle=N \sum_{i, j=1}^{N} \int d x_{1} \ldots d x_{N-1} d \xi d \eta v_{1}\left(x_{1}, \ldots, x_{j-1}, \xi, x_{j}, \ldots x_{N-1}\right) \times$ 


$$
\times v_{2}\left(x_{1}, \ldots, x_{i-1}, \eta, x_{i}, \ldots x_{N-1}\right) F_{\varepsilon}(\xi, \eta)
$$

where

$$
F_{\varepsilon}(\xi, \eta)=4 c^{2} \varepsilon^{d} \int d k f(\xi, k) g(k, \eta) \delta_{\varepsilon}(\xi-k) \delta_{\varepsilon}(k-\eta)
$$

Integrating over $x_{1}, \ldots x_{N-1}$, we obtain:

$$
\frac{1}{\varepsilon^{d}}\left\langle V_{1}, T_{1} V_{2}\right\rangle=\sum_{i, j=1}^{N} \int d \xi d \eta u_{i j}(\xi, \eta) F_{\varepsilon}(\xi, \eta)
$$

where $u_{i j}(\xi, \eta)$ are smooth functions. Using Lemma ??, we have:

$$
\lim _{\varepsilon \rightarrow 0} \frac{1}{\varepsilon^{d}}\left\langle V_{1}, T_{1} V_{2}\right\rangle=\sum_{i, j=1}^{N} \int d k u_{i j}(k, k) f(k, k) g(k, k)<\infty
$$

Thus, we conclude that

$$
\lim _{\varepsilon \rightarrow 0}\left\langle V_{1}, T_{1} V_{2}\right\rangle=0
$$

Hence,

$$
\underset{\varepsilon \rightarrow 0}{\mathrm{w} . \lim _{\boldsymbol{1}}} T_{1}=0
$$

Combining (??), (??), and (??), we obtain:

$$
\underset{\varepsilon \rightarrow 0}{\mathrm{w} . \lim _{\varepsilon \rightarrow 0}}\left(\left[B_{\phi, \varepsilon}^{-}, B_{\psi, \varepsilon}^{+}\right]\right)=\langle\phi, \psi\rangle
$$

Which is (??).

Let us prove (??). By Def. ??

$$
\left[N_{\phi, \varepsilon}, B_{\psi, \varepsilon}^{+}\right]=c \varepsilon^{d / 2}\left[N_{f_{\varepsilon}}, B_{g_{\varepsilon}}^{+}\right]
$$

Using Lemma ?? we have:

$$
c \varepsilon^{d / 2}\left[N_{f_{\varepsilon}}, B_{g_{\varepsilon}}^{+}\right]=2 c \varepsilon^{d / 2} B_{f_{\varepsilon} \diamond g_{\varepsilon}}^{+}=: T_{3}
$$

Let us choose any vectors $V_{1}, V_{2}$ of the form (??) with smooth $v_{1}$ and $v_{2}$. We have:

$$
\begin{aligned}
& \left\langle V_{1}, T_{3} V_{2}\right\rangle=2 c \varepsilon^{d / 2} \delta_{N, M+2} \sum_{i, j=1}^{M} \int d x_{1} \ldots d x_{M} d \xi d \eta \times \\
& \times v_{1}\left(x_{1}, \ldots, x_{i-1}, \xi, x_{i} \ldots, x_{j-1}, \eta, x_{j}, \ldots, x_{M}\right) v_{2}\left(x_{1}, \ldots x_{M}\right) G_{\varepsilon}(\xi, \eta)
\end{aligned}
$$


where

$$
G_{\varepsilon}=\int d k f(\xi, k) g(k, \eta) \delta_{\varepsilon}(\xi-k) \delta_{\varepsilon}(k-\eta)
$$

Integrating over $x_{1}, \ldots x_{M}$ we have:

$$
\frac{1}{\varepsilon^{d / 2}}\left\langle V_{1}, T_{3} V_{2}\right\rangle=\delta_{N, M+2} \sum_{i, j=1}^{M} \int d \xi d \eta u_{i j}(\xi, \eta) G_{\varepsilon}(\xi, \eta),
$$

where $u_{i j}$ are smooth functions. Applying Lemma ?? we have:

$$
\frac{1}{\varepsilon^{d / 2}}\left\langle V_{1}, T_{3} V_{2}\right\rangle=\delta_{N, M+2} \sum_{i, j=1}^{M} \int d k u_{i j}(k, k) f(k, k) g(k, k)<\infty
$$

Therefore, we conclude that

$$
\lim _{\varepsilon \rightarrow 0}\left\langle V_{1}, T_{3} V_{2}\right\rangle=0
$$

Eq. (??) can be proved similarly.

\section{The diagonal limit of the quadratic fields}

Definition 9 We say that a monomial $V$ in $B^{+}, B^{-}$, and $N$ is in normal form, if

$$
V=B_{f_{1}}^{+} \ldots B_{f_{k}}^{+} N_{g_{1}} \ldots N_{g_{m}} B_{h_{1}}^{-} \ldots B_{h_{n}}^{-}
$$

A polynomial $P$ is said to be in normal form if each of its monomials is in normal form.

Lemma 5 Let $V(\varepsilon)$ be a monomial in $B^{+}, B^{-}$, and $N$ of the form:

$$
V(\varepsilon)=X_{\phi_{n}, \varepsilon}^{n} \ldots X_{\phi_{2}, \varepsilon}^{2} X_{\phi_{1}, \varepsilon}^{1}
$$

where $X^{i}$ denotes either $B^{+}$, or $B^{-}$, or $N, X_{\phi_{i}, \varepsilon}=X_{S_{i}\left(\phi_{i}\right)_{\varepsilon}}, \phi_{i} \in$ $\mathcal{S}\left(\mathbb{R}^{d}\right)$. Then, independently on the embeddings $S_{i}$,

$$
\lim _{\varepsilon \rightarrow 0}\left\langle\psi_{0}, V(\varepsilon) \psi_{0}\right\rangle<\infty
$$

Moreover, replacing the product of the $X_{\phi_{i}, \varepsilon}^{i}$ by $X_{S_{i, 1}\left(\phi_{i, 1}\right)_{\varepsilon} \diamond \cdots \diamond S_{i, n_{i}}\left(\phi_{i, n_{i}}\right)_{\varepsilon}}^{i}$ the statement remains true. 
Proof. One can bring a monomial in $B^{+}, B^{-}$, and $N$ to the normally ordered form by applying the commutation relations (??-??).

Let us track the behavior of the coefficient functions during this process. In the beginning, one has $n$ coefficient functions $S\left(\phi_{1}\right)_{\varepsilon}, \ldots, S\left(\phi_{n}\right)_{\varepsilon}$.

Now consider a monomial $Y=Y_{f_{m}}^{m} \ldots Y_{f_{2}}^{2} Y_{f_{1}}^{1}$, where $Y^{i}$ denotes either $B^{+}$, or $B^{-}$, or $N$. Suppose we are going to apply the commutation relations to exchange $Y^{i}$ and $Y^{i-1}$. If $Y^{i}$ and $Y^{i-1}$ are $B^{-}$and $B^{+}$, then we have:

$$
Y=Z_{1}+Z_{2}+Z_{3}
$$

If $Y^{i}$ and $Y^{i-1}$ are $B^{-}$and $N$, or $N$ and $B^{+}$, then we have:

$$
Y=Z_{1}+Z_{2}
$$

where in $Z_{1}$ the coefficient functions change their order:

$$
Z_{1}=Y_{f_{m}}^{m} \ldots Y_{f_{i-1}}^{i-1} Y_{f_{i}}^{i} \ldots Y_{f_{2}}^{2} Y_{f_{1}}^{1}
$$

in $Z_{2}$ the coefficient functions are "coupled" by the $\diamond$-multiplication:

$$
Z_{2}=\varepsilon^{z} Y_{f_{m}}^{m} \ldots W_{f_{i-1} \diamond f_{i}} \ldots Y_{f_{2}}^{2} Y_{f_{1}}^{1}
$$

where $z$ is either $d$, or $0, W$ denotes either $B^{+}$, or $B^{-}$, or $N(W$ and $z$ depend on $Y^{i}$ and $Y^{i-1}$ ).

In $Z_{3}$ the coefficient functions are "traced out":

$$
Z_{3}=\varepsilon^{d} \operatorname{Tr}\left(f_{i} \diamond f_{i-1}\right) Y_{f_{m}}^{m} \ldots Y_{f_{2}}^{2} Y_{f_{1}}^{1}
$$

From this discussion we conclude that after a sequence of commutations a monomial is transformed into a finite sum of monomials. Each monomial term of the result contains all the functions of the original monomial. These test function functions may be combined by $\diamond$-multiplication. Such $\diamond$-product may be either the coefficient of an operator, or a factor under the trace. Moreover, each trace factor is accompanied by an $\varepsilon^{d}$ factor. Finally, an extra $\varepsilon^{n}$ factor, $n \geq 0$, can be present in some monomials.

If $V^{(N)}$ is a monomial in normal form and without constant term, then $\left\langle\psi_{0}, V^{(N)} \psi_{0}\right\rangle=0$. Therefore, only the constant terms survive. The most general form of a constant term is a finite product of the following traces:

$$
\varepsilon^{d+z} \operatorname{Tr}\left(S_{i_{1}}\left(\phi_{i_{1}}\right)_{\varepsilon} \diamond S_{i_{2}}\left(\phi_{i_{2}}\right)_{\varepsilon} \diamond \cdots \diamond S_{i_{r}}\left(\phi_{i_{r}}\right)_{\varepsilon}\right)
$$


where $z \geq 0$. Let us prove that Eq. (??) converges as $\varepsilon \rightarrow 0$. Indeed, denote $f_{k}=S_{i_{k}}\left(\phi_{i_{k}}\right)$. From Lemma ?? one has:

$$
\begin{gathered}
\lim _{\varepsilon \rightarrow 0} \varepsilon^{d+z} \int d k_{1} \ldots d k_{r} f_{1}\left(k_{1}, k_{2}\right) \ldots f_{r}\left(k_{r}, k_{1}\right) \delta_{\varepsilon}\left(k_{1}-k_{2}\right) \ldots \delta_{\varepsilon}\left(k_{r}-k_{1}\right)= \\
=\pi^{-r d / 2} \int d k f_{1}(k, k) f_{2}(k, k) \ldots f_{r}(k, k) \lim _{\varepsilon \rightarrow 0} \varepsilon^{z}= \\
=\pi^{-r d / 2} \int d k \phi_{i_{1}}(k) \phi_{i_{2}}(k) \ldots \phi_{i_{r}}(k) \lim _{\varepsilon \rightarrow 0} \varepsilon^{z}<\infty
\end{gathered}
$$

Thus, the vacuum expectation is equal to a finite sum of the form:

$$
\left\langle\psi_{0}, V(\varepsilon) \psi_{0}\right\rangle=\sum_{i=1}^{i_{0}} Z_{i}(\varepsilon)
$$

where each term $Z_{i}$ is a finite product:

$$
Z_{i}(\varepsilon)=\prod_{j=1}^{n_{i}} Z_{i, j}(\varepsilon)
$$

and the $Z_{i, j}(\varepsilon)$ are of the form (??). We proved that $\lim _{\varepsilon \rightarrow 0} Z_{i, j}(\varepsilon)<\infty$ therefore

$$
\lim _{\varepsilon \rightarrow 0}\left\langle\psi_{0}, V(\varepsilon) \psi_{0}\right\rangle=\sum_{i=1}^{i_{0}} \prod_{j=1}^{n_{i}} \lim _{\varepsilon \rightarrow 0} Z_{i, j}(\varepsilon)<\infty
$$

It is easy to check that if one replaces $X_{\phi_{i}, \varepsilon}^{i}$ by $X_{S_{i, 1}\left(\phi_{i, 1}\right)_{\varepsilon} \diamond \cdots \diamond S_{i, n_{i}}\left(\phi_{i, n_{i}}\right)_{\varepsilon}}^{i}$, then the proof is still correct.

Definition 10 Consider a monomial in $B^{+}$and $B^{-}$: Suppose the number of $B^{+}$and $B^{-}$operators is equal to $n$. A pair partition of this monomial is a sequence of $n$ pairs:

$$
\left\{\left(l_{1}, r_{1}\right),\left(l_{2}, r_{2}\right), \ldots,\left(l_{n}, r_{n}\right)\right\}
$$

such that

1. The set $\left\{l_{1}, r_{1}, l_{2}, r_{2}, \ldots, l_{n}, r_{n}\right\}$ is a permutation of the set $\{1,2, \ldots, 2 n\}$.

2. For any $i$ the $l_{i}$-th operator from the right is a $B^{-}$and the $r_{i}$-th operator from the right is a $B^{+}$.

3. For any $1 \leq i \leq n l_{i}>r_{i}$.

4. For any $1 \leq i<j \leq n, r_{i}<r_{j}$ 
Example: a pair partition of the monomial $B^{-} B^{-} B^{+} B^{-} B^{+} B^{+}$:

Theorem 2 Consider the vacuum expectation of the monomial

$$
\left\langle\psi_{0}, V(\varepsilon) \psi_{0}\right\rangle:=\left\langle\psi_{0}, X_{\phi_{n}, \varepsilon}^{n} \ldots X_{\phi_{2}, \varepsilon}^{2} X_{\phi_{1}, \varepsilon}^{1} \psi_{0}\right\rangle
$$

Then, independently of the embeddings,

1. If one of the $X$ 's is $N$, then $\left\langle\psi_{0}, V(\varepsilon) \psi_{0}\right\rangle \rightarrow 0$ as $\varepsilon \rightarrow 0$. Moreover, one can replace $X_{\phi_{i}, \varepsilon}^{i}$ by $X_{S_{i, 1}\left(\phi_{i, 1}\right)_{\varepsilon} \diamond \cdots \diamond S_{i, n_{i}}\left(\phi_{i, n_{i}}\right)_{\varepsilon}}^{i}$ and this statement is still valid.

2. If the number of $B^{+}$and $B^{-}$in $V$ is not the same, then $\left\langle\psi_{0}, V(\varepsilon) \psi_{0}\right\rangle \rightarrow$ 0 as $\varepsilon \rightarrow 0$.

3. If the number of $B^{+}$and $B^{-}$in $V$ is the same, then one has an analogue of the Wick theorem.

$$
\lim _{\varepsilon \rightarrow 0}\left\langle\psi_{0}, V(\varepsilon) \psi_{0}\right\rangle=\sum_{\text {All pairings }\left(l_{i}, r_{i}\right)} \prod_{\text {of } V}\left\langle\phi_{i}, \phi_{r_{n}}\right\rangle
$$

Proof. Let us prove the first statement. Suppose that one or more of the $X$ 's is $N$. Let us choose the rightmost $N$. We have

$$
\left\langle\psi_{0}, V(\varepsilon) \psi_{0}\right\rangle=\left\langle\psi_{0}, \ldots N_{\phi_{i}, \varepsilon} B_{\phi_{i-1}, \varepsilon}^{ \pm} \ldots B_{\phi_{1}, \varepsilon}^{ \pm} \phi_{0}\right\rangle
$$

Using Lemma ?? we have:

$$
\begin{aligned}
& \left\langle\psi_{0}, V(\varepsilon) \psi_{0}\right\rangle=\left\langle\psi_{0}, \ldots B_{\phi_{i-1}, \varepsilon}^{ \pm} \ldots B_{\phi_{1}, \varepsilon}^{ \pm} N_{\phi_{i}, \varepsilon} \phi_{0}\right\rangle+ \\
& +\sum_{j=1}^{i-1}\left\langle\psi_{0}, \ldots B_{\phi_{i-1}, \varepsilon}^{ \pm} \ldots\left( \pm 2 c^{2} \varepsilon^{d / 2} B_{S_{i}\left(\phi_{i}\right)_{\varepsilon} \diamond S_{j}\left(\phi_{j}\right)_{\varepsilon}}^{ \pm}\right) \ldots B_{\phi_{1}, \varepsilon}^{ \pm} \phi_{0}\right\rangle
\end{aligned}
$$


Since $N$ kills vacuum, the first term is always 0 . Using Lemma ?? we find that the second term $\left\langle\psi_{0}, T_{2}(\varepsilon) \psi_{0}\right\rangle$ satisfies

$$
\lim _{\varepsilon \rightarrow 0} \varepsilon^{-d / 2}\left\langle\psi_{0}, T_{2}(\varepsilon) \psi_{0}\right\rangle=\text { const }
$$

Hence, $\left\langle\psi_{0}, T_{2}(\varepsilon) \psi_{0}\right\rangle \rightarrow 0$ as $\varepsilon \rightarrow 0$. Using Lemma ?? it is easy to check that if one replaces $X_{\phi_{i}, \varepsilon}^{i}$ by $X_{S_{i, 1}\left(\phi_{i, 1}\right)_{\varepsilon} \diamond \cdots \diamond S_{i, n_{i}}\left(\phi_{i, n_{i}}\right)_{\varepsilon}}^{i}$, then the proof of Statement 1 is still correct.

The second statement is almost trivial. The number of creation and annihilation operators should be equal to obtain a non-zero vacuum expectation.

Let us prove the third statement. Note, that

$$
\left[B_{\phi, \varepsilon}^{-}, B_{\psi, \varepsilon}^{+}\right]=2 \operatorname{Tr} S(\phi)_{\varepsilon} \diamond S(\psi)_{\varepsilon}+4 N_{S(\phi)_{\varepsilon} \diamond S(\psi)_{\varepsilon}}
$$

But from Statement 1) of this Theorem it follows that any vacuum expectation of a monomial with at least one $N$ tends to 0 as $\varepsilon \rightarrow 0$. Therefore, we can bring $V$ to normal order using the effective relation

$$
\left[B_{\phi, \varepsilon}^{-}, B_{\psi, \varepsilon}^{+}\right]=2 \operatorname{Tr} S(\phi)_{\varepsilon} \diamond S(\psi)_{\varepsilon}
$$

and the vacuum expectation will be the same.

But (??) is the commutation relation between first order Bose creation and annihilation operators, and applying Wick's theorem we have

$$
\lim _{\varepsilon \rightarrow 0}\left\langle\psi_{0}, V(\varepsilon) \psi_{0}\right\rangle=\lim _{\varepsilon \rightarrow 0} \sum_{\text {All pairings }\left(l_{i}, r_{i}\right) \text { of } V} \prod_{i} \operatorname{Tr} S\left(\phi_{l_{n}}\right)_{\varepsilon} \diamond S\left(\phi_{r_{n}}\right)_{\varepsilon}
$$

and in the limit we get (??).

Corollary 1. (From Theorem ??) In the sense of correlators, the fields $B_{\phi, \varepsilon}^{+}, B_{\phi, \varepsilon}^{-}$converges to the Boson Fock field $a^{+}(\phi), a(\phi)$, and the field $N_{\phi, \varepsilon}$ converges to 0 .

$$
B_{\phi, \varepsilon}^{-} \rightarrow a(\phi), \quad B_{\phi, \varepsilon}^{+} \rightarrow a^{+}(\phi)
$$

Proof. This follows immediately from the the definition of the convergence in the sense of correlators (cf. Definition 3.1.1 of [?]) and the proof of statement 3) of the Theorem ??.

Now consider the following monomial in $B^{+}, B^{-}, a^{+}$and $a$ :

$$
W(\varepsilon)=a\left(\phi_{4}\right) B_{\phi_{3}, \epsilon}^{-} B_{\phi_{2}, \epsilon}^{+} a^{+}\left(\phi_{1}\right)
$$


We have:

$$
\begin{aligned}
\left\langle\psi_{0}, W(\varepsilon) \psi_{0}\right\rangle=\left\langle\psi_{0}, a\left(\phi_{4}\right)\left[B_{\phi_{3}, \epsilon}^{-},\right.\right. & \left.\left.B_{\phi_{2}, \epsilon}^{+}\right] a^{+}\left(\phi_{1}\right) \psi_{0}\right\rangle+ \\
& +\left\langle\psi_{0}, a\left(\phi_{4}\right) B_{\phi_{2}, \epsilon}^{+} B_{\phi_{3}, \epsilon}^{-} a^{+}\left(\phi_{1}\right) \psi_{0}\right\rangle
\end{aligned}
$$

Using Theorem ?? we find the limit of the first term:

$$
\begin{aligned}
\lim _{\varepsilon \rightarrow 0}\left\langle\psi_{0}, a\left(\phi_{4}\right)\left[B_{\phi_{3}, \epsilon}^{-}, B_{\phi_{2}, \epsilon}^{+}\right] a^{+}\left(\phi_{1}\right) \psi_{0}\right\rangle= \\
=\left\langle\phi_{2}, \phi_{3}\right\rangle\left\langle\psi_{0}, a^{+}\left(\phi_{4}\right) a\left(\phi_{1}\right) \psi_{0}\right\rangle=\left\langle\phi_{2}, \phi_{3}\right\rangle\left\langle\phi_{1}, \phi_{4}\right\rangle
\end{aligned}
$$

Since $\left\langle\psi_{0}, a_{k_{1}} a_{k_{2}}^{+} a_{k_{3}}^{+} a_{k_{4}} a_{k_{5}} a_{k_{6}}^{+} \psi_{0}\right\rangle=0$, the second term is equal to 0 . Therefore, we conclude that

$$
\lim _{\varepsilon \rightarrow 0}\left\langle\psi_{0}, W(\varepsilon) \psi_{0}\right\rangle=\left\langle\phi_{2}, \phi_{3}\right\rangle\left\langle\phi_{1}, \phi_{4}\right\rangle
$$

Therefore, one can't evaluate $\lim _{\varepsilon \rightarrow 0}\left\langle\psi_{0}, W(\varepsilon) \psi_{0}\right\rangle$ by substituting $a^{+}(\phi)$ for $B_{\phi, \varepsilon}^{+}$and $a(\phi)$ for $B_{\phi, \varepsilon}^{-}$in $W(\varepsilon)$ and removing the limit, because

$$
\left\langle\psi_{0}, a_{\phi_{4}} a_{\phi_{3}} a_{\phi_{2}}^{+} a_{\phi_{1}}^{+} \psi_{0}\right\rangle=\left\langle\phi_{2}, \phi_{3}\right\rangle\left\langle\phi_{1}, \phi_{4}\right\rangle+\left\langle\phi_{2}, \phi_{4}\right\rangle\left\langle\phi_{1}, \phi_{3}\right\rangle
$$

In this sense we say that as $\varepsilon \rightarrow 0 B_{\phi, \varepsilon}^{ \pm}$converges to the Boson Fock field, defined in a new Hilbert space

\section{$7 \quad$ Independence on regularization.}

The goal of the present section is to show that

- The result of Theorem ?? doesn't depend on the $\delta$-function regularization (??).

- The renormalization factor $\varepsilon^{d / 2}$ in Def. ?? is uniquely determined.

Definition 11 We call a sequence of functions $\omega_{\varepsilon}(x), 0<\varepsilon<\varepsilon_{0}$, reasonable if

$$
\omega_{\varepsilon}(x)=\varepsilon^{A-d} \Omega\left(\frac{x}{\varepsilon}\right)
$$

where $\Omega \in \mathcal{S}\left(\mathbb{R}^{d}\right)$ is such that

$$
\int \Omega(x) d x=1
$$


Remark. One can see, that if $A=0$ then $\omega_{\varepsilon}$ is a delta-sequence in $\mathbb{R}^{d}$.

Lemma 6 Suppose $\omega_{\varepsilon}(x)$ is a reasonable sequence and

$$
0 \neq \lim _{\varepsilon \rightarrow 0} \int \omega_{\varepsilon}^{2}(x) d x<\infty
$$

in the sense that the limit exists and the inequalities hold. Then, $A=\frac{d}{2}$.

Proof. We have:

$$
\begin{gathered}
\int \omega_{\varepsilon}^{2}\left(x_{1}, \ldots x_{d}\right) d x_{1} \ldots d x_{d}= \\
=\int \varepsilon^{2 A-2 d} \Omega^{2}\left(\frac{x_{1}}{\varepsilon}, \ldots \frac{x_{d}}{\varepsilon}\right) d x_{1} \ldots d x_{d}= \\
=\varepsilon^{d+(2 A-2 d)} \int \Omega^{2}\left(\frac{x_{1}}{\varepsilon}, \ldots \frac{x_{d}}{\varepsilon}\right) d\left(\frac{x_{1}}{\varepsilon}\right) \ldots d\left(\frac{x_{d}}{\varepsilon}\right)= \\
=\varepsilon^{2 A-d} \int \Omega^{2}\left(\xi_{1}, \ldots, \xi_{d}\right) d \xi_{1} \ldots d \xi_{d}
\end{gathered}
$$

Since $\Omega \in \mathcal{S}\left(\mathbb{R}^{d}\right)$ the integral exists and not equal to zero. (Otherwise, $\Omega(x)=0$ almost everywhere, and $\left.\int \Omega(x) d x=0\right)$. Hence the limit in (??) always exists and the only possibility for it to $b e \neq 0, \infty$ is that $A=\frac{d}{2}$.

Now suppose that instead of Def. ?? we define:

$$
\begin{aligned}
& B_{\phi, \varepsilon}^{+}:=B_{S(\phi)\left(p_{1}, p_{2}\right) \omega_{\varepsilon}\left(p_{1}-p_{2}\right)}^{+} \\
& B_{\phi, \varepsilon}^{-}:=B_{S(\phi)\left(p_{1}, p_{2}\right) \omega_{\varepsilon}\left(p_{1}-p_{2}\right)}^{-}
\end{aligned}
$$

where $\omega_{\varepsilon}(x)$ is a reasonable sequence. We require that $\lim _{\varepsilon \rightarrow 0}\left[B_{\phi, \varepsilon}^{-}, B_{\psi, \varepsilon}^{+}\right]$ exists and is $\neq 0, \infty$. Then, repeating the proof of Theorem ?? we find that the scalar part of the commutator is equal to

$$
T_{2}:=\int d p_{1} d p_{2} f\left(p_{1}, p_{2}\right) g\left(p_{1}, p_{2}\right) \omega_{\varepsilon}^{2}\left(p_{1}-p_{2}\right)
$$

Choosing $f$ and $g$ such that $f\left(p_{1}, p_{2}\right)=f_{0}\left(p_{1}+p_{2}\right), g\left(p_{1}, p_{2}\right)=g_{0}\left(p_{1}+\right.$ $\left.p_{2}\right)$ in some neighborhood of the diagonal, we find that a necessary condition for the convergence is:

$$
\int \omega_{\varepsilon}^{2}(p) d p<\infty
$$


From Lemma ?? we know that $A=\frac{d}{2}$. Therefore

$$
\omega_{\varepsilon}(x)=\varepsilon^{d / 2} \frac{1}{\varepsilon^{d}} \Omega\left(\frac{x}{\varepsilon}\right)=\frac{1}{\varepsilon^{d / 2}} \Omega\left(\frac{x}{\varepsilon}\right)
$$

Now we are going to prove that $T_{1}$, the number part of the correlator, tends to 0 as $\varepsilon \rightarrow 0$. Again, repeating the proof of Theorem ??, we find that for any number vectors $V_{1}, V_{2}$

$$
\left\langle V_{1}, T_{1} V_{2}\right\rangle=\sum_{i, j=1}^{N} \int d \xi d \eta u_{i j}(\xi, \varepsilon) F_{\varepsilon}(\xi, \eta)
$$

where

$$
F_{\varepsilon}(\xi, \eta)=\int d k f(\xi, k) g(k, \eta) \omega_{\varepsilon}(\xi-k) \omega_{\varepsilon}(k-\eta)
$$

Changing variables, we have:

$$
\left\langle V_{1}, T_{1} V_{2}\right\rangle=\sum_{i, j=1}^{N} \int d x d y d z \omega_{\varepsilon}(x) \omega_{\varepsilon}(y) u_{i j}(x, y, z)
$$

Note that $\delta_{\varepsilon}(x)=\frac{1}{\varepsilon^{d}} \Omega\left(\frac{x}{\varepsilon}\right)$ is a delta-sequence. Therefore, $\omega_{\varepsilon}(x)=$ $\varepsilon^{d / 2} \delta_{\varepsilon}(x)$ and for any $f \in \mathcal{S}^{\prime}\left(\mathbb{R}^{d}\right)$ we have:

$$
\lim _{\varepsilon \rightarrow 0} \int \omega_{\varepsilon}(x) f(x) d x=\lim _{\varepsilon \rightarrow 0} \varepsilon^{d / 2} \int \delta_{\varepsilon}(x) f(x) d x=\lim _{\varepsilon \rightarrow 0} \varepsilon^{d / 2} f(0)=0
$$

Therefore, $\lim _{\varepsilon \rightarrow 0}\left\langle V_{1}, T_{1} V_{2}\right\rangle=0$.

We summarize this result as a theorem:

Theorem 3 Suppose $B^{+}$and $B^{-}$are given by (??, ??), where $\omega_{\varepsilon}$ is a reasonable sequence and

$$
\lim _{\varepsilon \rightarrow 0}\left[B_{\phi, \varepsilon}^{-}, B_{\psi, \varepsilon}^{+}\right]
$$

converges. Then, this limit is a scalar and the renormalization constant must be $A=d / 2$.

\section{Conclusions}

We have defined regularized and renormalized quadratic fields that tend to be localized near the diagonal (Def. ??). We have proved 
that, when the regularization tends to become sharp, these fields tend to a boson Fock field (Corollary 1 of Theorem ??). We have also proved that our result doesn't depend on the choice of the regularization within a rather wide class of delta-sequences, and that the renormalization constant is uniquely determined (Theorem ??).

\section{Acknowledgements}

The authors are grateful to I.Aref'eva and I.Volovich for helpful discussions.

The research activities of L.A. was partially supported by the European Union Research Training Network: "Quantum Probability with Applications to Physics, Information Theory and Biology". R.R. was partially supported by the RFFI grant 02-01-01084 and the grant 1542.2003.1 for scientific schools.

\section{References}

[AcLuVo02] L. Accardi, Y.G. Lu, I.V. Volovich: Quantum Theory and Its Stochastic Limit. Springer Verlag (2002)

[AcLuVol99] Accardi L., Lu Y.G., Volovich I.V.: White noise approach to classical and quantum stochastic calculi, Lecture Notes of the Volterra International School of the same title, Trento, Italy, 1999, Volterra Preprint N. 375 July (1999)

[AcVol97] L. Accardi, I.V. Volovich: Quantum White Noise with singular Non-Linear Interaction, in: Developments of InfiniteDimensional Noncommutative Analysis, N. Obata (ed.) RIMS Kokyuroku 1099 Publications of the Research Institute for Mathematical Sciences, Kyoto (1999) 6170, Volterra preprint N. 278 (1997) quant-ph/9704029

[AcSk99b] Accardi L., Skeide M.: On the relation of the Square of White Noise and the Finite Difference Algebra, Infinite dimensional analysis, quantum probability and related topics 3 (2000) 185-189 Volterra Preprint N. 386 (1999)

[AcFrSk00] Accardi L., Franz U., Skeide M.: Renormalized squares of white noise and other non- Gaussian noises as Levy processes on real Lie algebras, Comm. Math. Phys. 228 (2002) 123-150 Preprint Volterra, N. 423 (2000)

[AcHiKu01] Accardi L., Hida T., Kuo H.H.: The Itô table of the square of white noise, Infinite dimensional analysis, quantum 
probability and related topics, 4 (2) (2001) 267-275 Preprint Volterra, N. 459 (2001)

[AcBou01f] Accardi L., Boukas A., Kuo H.-H.: On the unitarity of stochastic evolutions driven by the square of white noise, Preprint Volterra N. 464 (2001) Infinite Dimensional Analysis, Quantum Probability, and Related Topics 4 (4) (2001) 1-10

[AcAmFr02] Accardi L., Amosov G., Franz U.: Luigi Accardi, Grigori Amosov, Uwe Franz: Second quantized automorphisms of the renormalized square of white noise (RSWN) algebra IDA-QP 7 (2) (2004) Preprint Volterra (2002)

[BogSh80] Bogoliubov N. N., Shirkov D. V.: Introduction to the theory of quantized fields, John Wiley \& Sons, 1980.

[Ber66] F. A. Berezin: The Method of Second Quantization, Academic Press, Pure Appl. Phys. 24 (1966)

[GlJa81] Quantum physics. A functional integral point of view / Glimm James, Jaffe Artur. - 1981

[Lytv04] Eugene Lytvynov: The square of white noise as a Jacobi field, submitted to IDA-QP (2004)

[Sega70a] Segal I.E.: Transformations in Wiener space and squares of quantum fields, Adv. in Math. 4 (1970) 91-108

[Shal62] Shale D.: Linear symmetries of free boson fields, Trans. Amer. Math. Soc. 103 (1962) 149-167

[Snia99] Śniady P.: Quadratic bosonic and free white noises, Commun. Math. Phys: 211 (3) (2000) 615-628 Preprint (1999)

[Vlad71] Vladimirov, V. S. Equations of Mathematical Physics. New York: Dekker, 1971. 